\title{
Extremophiles
}

July 2009, Volume 13, Number 4, Pages 583-594

Archimer, archive institutionnelle de l'Ifremer http://dx.doi.org/10.1007/s00792-009-0261-3

(c) 2009 Springer. Part of Springer Science+Business

Media

The original publication is available at http://www.springerlink.com

\section{Cultivating the uncultured: limits, advances and future challenges}

\author{
Karine Alain ${ }^{1}$ and Joël Querellou ${ }^{2, *}$ \\ ${ }^{1}$ UMR6197, Laboratoire de Microbiologie des Environnements Extrêmes, IUEM, Technopôle Brest-Iroise, 29280 \\ Plouzané, France \\ ${ }^{2}$ UMR6197, Laboratoire de Microbiologie des Environnements Extrêmes, Ifremer, Centre de Brest, Pointe du \\ diable, BP 70, 29280 Plouzané, France \\ *: Corresponding author : J. Querellou, email address : jquerell@ifremer.fr
}

\begin{abstract}
:
Since the invention of the Petri dish, there have been continuous efforts to improve efficiency in microbial cultivation. These efforts were devoted to the attainment for diverse growth conditions, simulation of in situ conditions and achievement of high-throughput rates. As a result, prokaryotes catalysing novel redox reactions as well as representatives of abundant, but not-yet cultured taxa, were isolated. Significant insights into microbial physiology have been made by studying the small number of prokaryotes already cultured. However, despite these numerous breakthroughs, microbial cultivation is still a low-throughput process. The main hindrance to cultivation is likely due to the prevailing lack of knowledge on targeted species. In this review, we focus on the limiting factors surrounding cultivation. We discuss several cultivation obstacles, including the loss of microbial cellcell communication following species isolation. Future research directions, including the refinement of culture media, strategies based on cell-cell communication and high-throughput innovations, are reviewed. We further propose that a combination of these approaches is urgently required to promote cultivation of uncultured species, thereby dawning a new era in the field.
\end{abstract}

Keywords: Culture - Isolation - Metabolism - Microbial community - Cell-cell communication - Highthroughput cultivation 


\section{Introduction}

Today, 1.8 million eukaryotic species are recognized, while estimates indicate that our planet hosts 5 to 10 -fold more species.. Until now, only 7031 prokaryotic species have been described (and validated by the International Committee on Systematics of Prokaryotes) in the one hundred and twenty years, since the invention of the Petri dish (Achtman \& Wagner, 2008), even though we now realize that prokaryotes represent "the unseen majority" (Whitman et al., 1998). The lack of an extensive and accurate picture of the microbial diversity is partly due to a lack in technical advances in the microbiology cultivation field. . Over the past three decades, molecular biology was an enormous driving force in microbiology in uncovering the microbial diversity . Many new candidate divisions are now recognized due to $16 \mathrm{~S}$ rRNA sequence-based approaches and environmental metagenomics (Curtis et al., 2002). These findings exposed a gap between the known phyla and those possessing cultured representatives (Fig. 1). In 1987, when much of our knowledge derived from pure culture techniques, all the phyla known possessed cultured representatives. Twenty years later, of the 100 bacterial phyla identified only 30 possess a cultivated representative (Achtman \& Wagner, 2008). The number of phylum-level divisions possessing cultured representatives has increased at a linear and constant rate over the last two decades. However, this increase was greatly augmented with the emergence of molecular-based approaches. This augmentation is seen when candidate divisions are included in these calculations (Fig. 1)..

From a quantitative point of view, the advances made by culture-dependent approaches may appear trivial, especially since only $0.1 \%$ of the existing prokaryotes have been cultured so far. Yet, this quantitative approach is reductive since the past 20 years of microbial cultivation have led to unprecedented advances in our knowledge of the microbial world. 
Molecular ecology and metagenomics have increased significantly our knowledge of the genetic diversity and have led to interesting hypotheses (Hugenholtz \& Tyson, 2008). The advanced techniques have also revealed how far we are from measuring the full extent of genetic diversity encoded by microbial life (Hugenholtz \& Tyson, 2008; Pignatelli et al., 2008). Considering that many of the genes stored in the databases have unknown functions or are incorrectly annotated, it is probable that metagenomes alone will not offer sufficient knowledge to cultivate all organisms. There is evidence that many of the candidate divisions revealed by molecular approaches, and known only from molecular signatures, represent a significant fraction of the microbial diversity. Some members of these 'not-yet-cultured' taxa are probably key ecological players. Today, one of the main challenges for microbiologists is to develop strategies to cultivate this uncultured majority. A comprehensive understanding of biology and ecology of prokaryotes will require cultivation. Therefore, it is not surprising to observe an increasing interest for the field of microbial cultivation (Leadbetter, 2003).

The aim of this review is to provide an overview of the new cultivation-based approaches while documenting their limitations and outcomes. Further, we highlight how cultivation has led to valuable advances in our understanding of microbial physiology and identify the future challenges for microbiologists in the microbial cultivation field. Lastly, a discussion is provided on the technical developments that may drive innovative research in the near future.

\section{I- Review on some cultivation successes}

Prokaryotic growth necessitates an energy source (light or chemical compounds), nutrients and proper physicochemical conditions. Challenges for the microbiologist are to identify required nutrients, to provide them in the growth medium in the appropriate concentrations to sustain the microbial growth, and to avoid the co-precipitation of the introduced chemicals. Since different organisms require a different set of nutrients in varying concentrations and 
forms, the design of growth media remains a difficult task. Conversely, the intrinsic selectively of any growth medium imposes limitations on the type, diversity and number of prokaryotes recovered from the natural environment. This phenomenon is known as the "great plate count anomaly” (Staley \& Konopka, 1985). Indeed, there is a difference of several orders of magnitude between colony counts on laboratory medium and total numbers of prokaryotic cells present in natural environments. Only a minor fraction of the naturally occurring microbial community is recovered by conventional selective media (Skinner et al., 1952; Amann et al., 1995). Depending on the nature of the samples, the cultivation efficiency of active cells by standard plating technique is estimated between $0.001 \%$ and $1 \%$ (Kogure et al., 1979; Staley \& Konopka, 1985; Amman et al., 1995). Thus, cultured microorganisms do not reflect the functional and phylogenetic diversity present within any natural habitat.

Two main strategies are used for the isolation of pure cultures in microbiology. In both strategies, enrichment culture is performed as a first step. The first strategy aims to isolate colonies by repetitive streaking on solid medium (or alternatively by performing pour plates or agar shake tubes), while the second strategy aims to isolate cells following repeated series of dilutions in liquid medium. These classical approaches have led to the isolation of a large number of strains belonging to taxa with few or no representatives in pure culture (Janssen et al., 1997; Joseph et al., 2003). Nevertheless, the enrichment and pure culture isolation strategies often select for opportunistic fast-growing organisms also called lab weeds. In nutrient-rich artificial media, the community members with ' $r$ '-strategy, or fast-growers, often overgrow and outcompete the naturally abundant ' $\mathrm{K}$ '-strategists (Watve et al., 2000). Consequently, these conventional culture-dependent approaches do not reflect the actual microbial communities (Amann et al., 1995). Certain taxa are still severely under-represented in pure cultures (Hugenholtz et al., 1998). As direct consequence, most of our current 
knowledge of the nutrition, physiology and biochemistry of prokaryotes is based on easily cultivable organisms.

In recent years, novel cultivation strategies were developed to overcome these limitations. Schematically, they are classified into four categories (Fig. 2).

\section{1- Refinement of standard cultivation strategies}

Different studies have demonstrated that a fraction of the "not-yet-cultured" groups of prokaryotes can be grown by the refinement of classical approaches. Changes in the media formulations, including the use of non-traditional electron donors, electron acceptors and carbon sources have proven efficient in recovery of uncultured taxa (Köpke et al., 2005). Diversification of the media and multiplication of culture conditions are simple methods to by-pass approaches that are selective by nature. For instance, the cultivable fraction from coastal subsurface sediments was shown to yield a higher number and diversity of isolates when culture collections were performed with diverse electron acceptors and carbon sources (Köpke et al., 2005). The qualitative composition of carbon sources is also a determinant factor for cultivation efficiency .. As shown with seawater samples from the North Sea, media prepared using several different carbon sources and complex compounds yield higher number and more diverse isolates than similar media with only one carbon source. These isolates obtained with a single substrate belonged almost exclusively to the Gammaproteobacteria while representatives of four other classes grew on complex media (Uphoff et al., 2001). In recent years, it was shown that novel redox reactions, catalysed by specific ecological communities of prokaryotes, can be identified within enrichment cultures onto non-conventional media. Many novel physiotypes can be isolated using this method. Significant advances were made in the field of the anaerobic degradation of hydrocarbons. For example, different microbial consortia of Archaea and Bacteria, which couple the 
anaerobic oxidation of methane to sulphate or nitrate reduction, were enriched from anoxic marine or freshwater sediments. These findings enhanced our understanding of the global biological cycles (Nauhaus et al., 2002; Raghoebarsing et al., 2006). Moreover, hydrocarbondegrading bacteria were identified using refined media containing only hydrocarbon/carbon energy sources and nitrate, iron or sulphate as electron acceptors. These concurrent studies led to a better understanding of the biochemistry and energetics of anaerobes. Several biochemical mechanisms involved in the activation of some of these chemically non-reactive compounds have been identified (Lovley and Lonergan, 1990; Rabus et al., 1993; Galushko et al., 1999; Coates et al., 2001; Widdel et al., 2007). However, hydrocarbon-degrading capacities, activation mechanisms and species or ecological guilds, which are involved in hydrocarbon degradation, remain to be discovered. For example, a microbiological anaerobic oxidation of methane with iron (III) or manganese (IV) as a terminal electron acceptor is unknown, yet this reaction is thermodynamically possible (Raghoebarsing et al., 2006). The enrichment culture with anoxic ditch sediment, discovered in 1999 by Zengler and coworkers, was another growth-supporting reaction of relevance. This team demonstrated that the conversion of long-chain alkanes to methane, under strictly anoxic conditions, is biologically performed by an ecological guild assumed to be acetogenic syntrophic bacteria associated to acetoclastic and hydrogenotrophic methanogenic archaea (Zengler et al., 1999). The discovery of this process in nature might help to understand the terminal degradation of organic matter in areas of deep and old marine sediments where sulphate is depleted. Another novel physiotype recently identified due to advances in cultivation and isolation was an autotrophic anaerobe, which couples the oxidation of phosphite (III) to sulphate reduction (Schink and Friedrich, 2000). These novel physiotypes along with the newly recognized biological redox reactions are only a few of the several examples of the significant advances 
made in uncovering the microbial diversity through the enhancement of cultivating techniques.

Some recent successes in improving traditional cultivation methods include the following. The use of relatively low concentration of nutrients to increase the cultivability and to improve the recovery of prokaryotes from different types of natural samples (Button et al., 1993; Janssen et al., 1997; Watve et al., 2000; Connon and Giovannoni, 2002; Rappé et al., 2002; Sangwan et al., 2005). The use of increased incubation periods to allow for the development of strains from rarely isolated taxa (Sait et al., 2002; Stevenson et al., 2004; Davis et al., 2005; Sangwan et al., 2005; Stott et al., 2008) and, the addition of signalling compounds known to mediate communication between bacteria (Bruns et al., 2002; Bruns et al., 2003). Moreover, other less-documented approaches also yielded new isolates. These included: the use of gellan gum (phytagel) as a gelling reagent instead of agar (Tamaki et al., 2005; Stott et al., 2008); the decrease in inoculum size (Davis et al., 2005); the addition of electron transporters to the culture media (Stevenson et al., 2004); the addition of enzymes to cope with reactive oxygen species (Stevenson et al., 2004); the addition of inhibitors of undesired organisms (Leadbetter et al., 1999); and, the combination of an unusual energy source with antibiotics to exclude Bacteria (Könneke et al., 2005). Finally, sophisticated single-cell isolation tools allowing for the manipulation of a targeted cell from a mixed community (with a micro-capillary tube or with 'optical tweezers') have been developed (Huber et al., 1995; Fröhlich and König, 2000; Huber et al., 2000). The principal limitations of these single-cell isolation strategies include their labour intensive requirements and the determination of the suitable growth conditions to cultivate prokaryotic cells of unknown metabolism and systematic affiliation. The identification of a cell of interest among a complex community in absence of clear distinctive morphological features is also challenging. Altogether, these limitations account for the lack in isolating numerous undiscovered strains. 


\section{2- Cultures in situ or cultures in simulated natural conditions}

Often, the laboratory conditions poorly mimic the natural environmental conditions. Therefore, strategies aimed at simulating natural conditions or culturing in situ have been proven efficient. Schematically, two types of "in situ colonization devices" have been developed: the diffusion chambers and the carriers (of organic or inorganic nature) (Kaeberlein et al., 2002; Ferrari et al., 2005; Yasumoto-Hirose et al., 2006; Bollmann et al., 2007). Diffusion chambers are apparatuses equipped with filter membranes which restrict the movement of cells in the chamber. They allow for the removal of low-molecular weight inhibitory end-products, as well as the exchange of chemicals between the chamber and the environment, thereby making high density cultivation possible (Pörtner and Märkl, 1998; Kaeberlein et al., 2002). Different types of membrane-based systems have been developed to grow microbial communities directly in the natural habitats (Kaeberlein et al., 2002; Plugge and Stams, 2002; Ferrari et al., 2005; Bollmann et al., 2007; Ferrari et al., 2008). Uncultured bacteria from soil, marine or activated sludge were grown in diffusion chambers. This led to the hypothesis that in situ cultivation of environmental prokaryotes in diffusion chambers either enriches sufficiently the strains for their subsequent isolation onto classical solid media or conditions them for growth under otherwise prohibitive in vitro conditions (Bollmann et al., 2007). Interestingly, slow-growing organisms were cultivated using this method.

In natural ecosystems, many prokaryotes live attached to surfaces. This is well known for microbes living in sediments, soils, or in association with eukaryotes. It is less recognized for microbes living in aquatic habitats, where free-living forms were supposed to be dominant, but other associations, with various interfaces, exist. The attached existence provides several advantages for the prokaryotes. Attached cells escape grazing better than their free-living neighbours. Attachment also allows cells to develop metabolic inter-relations, resistance to 
different stressesand better access to adsorbed substrates (Schink, 1999). In situ colonization carriers are useful tools to overcome cultivation limits induced by attachment of prokaryotes to solid surfaces. Several publications describe the deposition in natural ecosystems of different carriers such as glass, ceramic, titanium devices, porous inorganic substrates or polyurethane foams (Araki et al., 1999; Alain et al., 2004; Yasumoto-Hirose et al., 2006). In situ collectorscoated with selective substrates, are effective for the selective enrichment of targeted prokaryotes (Yasumoto-Hirose et al., 2006). In addition, specialized techniques simulating one or several important spatial or physical parameters allow the cultivation of novel physiotypes. For instance, gradient systems (Nelson and Jannasch, 1983; Emerson and Moyer, 1997), high pressure reactors (Marteinsson et al., 1999; Alain et al., 2002), flowthrough devices (Houghton et al., 2007) and gas-lift reactors (Postec et al., 2005; Postec et al., 2007) were successfully used. . Pressure is also an essential parameter given that highpressure environments occupy the largest fraction of the known biosphere.This parameter was often neglected in microbial cultivation. However, its effects on microbial life are as important as those of temperature or salinity. Pressure acts upon physiology and upon biochemical reactions. Thus, these types of reactors are effective to grow microbial strains from high pressure habitats. However, only few piezophilic prokaryotes have been enriched or isolated under elevated pressures (i.e. Yayanos et al.,1979, 1981; Marteinsson et al.,1999; Alain et al., 2002; Houghton et al., 2007). Recently, the first obligate piezophilic and hyperthermophilic archaeon (Pyrococcus sp. strain CH1) was isolated from the deepest hydrothermal vent field explored so far, using a high pressure reactor (Zeng et al., in press). Finally, different types of bioreactors are used to reproduce diverse environmental conditions.The physical and chemical conditions. of seafloor hydrothermal systems are among the most difficult to reproduce in laboratories, considering the multiplicity of in situ physical and chemical gradients. Gas-lift reactors and flow-through devices are powerful 
tools to simulate hydrothermal vent in situ conditions (Postec et al., 2005; Postec et al., 2007; Houghton et al., 2007). Flow-through devices allow for continuous cultures under in situ temperature, pressure and fluid flow.They make possible the simulation of the deep-sea vent changes of fluid chemistry (Houghton et al., 2007). Both systems allow for continuous enrichment culturing under controlled conditions. Significant fractions of microbial communities were grown using these systems (Postec et al., 2007).

\section{3- Cultures of microbial communities}

The cultivation methods allowing for the growth of mixed populations offer great potential to cultivate not-yet cultivated organisms.Indeed, in natural environments, most organisms live as a part of a community in which distinct cells work in concert and communicate either by trading metabolites, by exchanging dedicated signalling molecules, or by competition for limited resources (West et al., 2007; Nadell et al., 2009). These relationships, in addition to complex cell-cell communications, are hardly reproducible in monocultures. This "in group" lifestyle, in biofilm or multi-cellular assemblage, is thought to generate robustness to nutritional, biotic and abiotic changes by providing a capacity of adaptation to environmental fluctuations. It is critical for microbial ecology and evolution. A striking example is biofilm, in which cell-cell communication determines biofilm structure, maturation and microbial niche construction thereby optimizing microbial survival and reproduction. Mixed microbial assemblages have also the capacity to perform multiple-step functions that are often not possible for individual strains or species. Examples are the degradation of cellulose or the methanogenic conversion of complex organic matter (Brenner et al., 2008). In some cases, the cooperation within the microbial community is based on sharing metabolic intermediates, micronutrients (e.g. vitamins) or chelating agents that either assist or compromise the growth of other community members. When identified, such facultative dependencies can be 
reproduced experimentally by supplying the medium with these micronutrients or cosubstrates. Besides facultative associations, syntrophic associations are often compulsory and this interdependence cannot easily be by-passed or suppressed by the addition of factors to the media (Schink, 1999). Many syntrophic associations are explained by unfavourable energetic conditions. . Both facultative and syntrophic associations are widespread in natural habitats. Consequently, approaches based on community cultures are effective methods to grow facultative associations and syntrophic organisms. Using community culture approaches in addition to dialysis membrane reactors, thermophilic syntrophic anaerobic glutamatedegrading consortia from anaerobic sludge have been successfully enriched (Plugge and Stams, 2002). Similarly, batch reactors operating under anaerobic-aerobic cycling conditions have allowed the enrichment of mixed microbial sludge communities (Crocetti et al., 2002).

\section{4- High-throughput automatable microbial culture formats}

The past decade was marked by the emergence of high-throughput cultivation methods (Connon and Giovannoni, 2002; Zengler et al., 2002; Zengler et al., 2005; Ingham et al., 2007). Several advances in high-throughput culture formats have originated from industrial endeavours. Indeed, the myriad of organisms inhabiting our planet represents a tremendous reservoir of bio-molecules for pharmaceutical, agricultural, industrial and chemical applications. While culture-independent recombinant approaches are used to screen novel molecules and enzymes from natural samples, cultivation of organisms greatly simplifies such studies and allows the use of a strategy biased for the desired phenotype.

A very efficient high-throughput cultivation method resulted from modifying the pioneering work, called extinction cultures, of Button and colleagues (Button et al., 1993). Briefly, extinction culturing requires dilutions of natural communities to a low number of cells ( 1 to 5 cells per ml) in natural environmental water followed by their incubation in defined 
conditions. Following incubation, growth is measured. The initial procedure . was refined by Giovannoni and colleagues to increase high throughput rates by using microtiter plates and by fluorescence microscopy screening (Connon and Giovannoni, 2002; Stingl et al., 2007). This improved technique resulted in better sensitivity and cultivation efficiency. This technique allows notably for the growth of slow-growers. It also allowed for the isolation of the first representatives of two bacterial clades: the SAR11 clade, a ubiquitous alpha-proteobacterial lineage found abundantly in marine pelagic environment (Rappé et al., 2002) and the OM43 clade (Connon and Giovannoni, 2002).

Another technology for massive cultivation of prokaryotes was developed recently. This highthroughput approach consists in the encapsulation of cells in gel microdroplets (GMDs) incubated in a single column for long intervals of time under low nutrient flux conditions. Micro-capsules are subsequently sorted by flow cytometry.. This technique is applied to samples from different habitats and provides more than 10000 bacterial and fungal isolates per natural sample (Zengler et al., 2005). To our knowledge, this technology, which allows the development and detection of micro-colonies (20 to 100 cells), has never been used for anaerobes. It is advantageous as cell-containing micro-droplets are grown together in capsules allowing for the exchange of signal compounds and metabolites between cells from different micro-colonies but originating from the same natural community. Using the microencapsulation approach, members of numerically abundant clades were isolated. Although very promising, this technique is not yet easily implemented in microbiology laboratories (Zengler et al., 2002; Zengler et al., 2005).

Finally, a multiwell microbial culture-chip was recently developed by a team of microbiologists, nanotechnologists and micro-engineering experts (Ingham et al., 2007). This micro-Petri dish, is composed of a unique porous ceramic subdivided into millions of compartments in which cultures can be separately grown. The growth of microorganisms is 
sustained by the nutrients diffused through a porous membrane. The innovation lies in the conception of the chip that is composed of micron-scale wells, is readily manufactured, affordable and easy-to-use in microbiology laboratories not equipped with micromanipulator. This system combines automation and miniaturisation, prerequisites for modern microbiology.

All approaches described in this chapter aimed to optimize prokaryote cultivation efficiency (fig. 2). Overall, these studies allow for the isolation of numerous novel species and permit major breakthroughs in cultivation. Despite continuous efforts, culture dependent approaches undergo changes characterized by gradual improvements rather than by a radical paradigm shift. It is noteworthy that the so-called "Moore Law" in microbiology (Gefen and Balaban, 2008) applies to the volume used in culture and not to the output in number of novel species described.

\section{II-Why do so many microbes resist cultivation?}

Despite extensive studies on ecosystems where sampling is conducted, the conditions used in classical cultivating conditions are often far from endogenous abiotic and biotic conditions required for microbial growth. Thus, it is not surprising that only a small fraction of the whole microbial repertoire has been cultured so far.

The lack of efficient cultivation techniques stems from many factors that largely remain unknown. These factors include limited knowledge of (i) the diverse organisms, (ii) the chemistry of the natural habitats, (iii) the natural biotic and abiotic interactions and (iv) the global functioning of the ecosystems at microbial level. Cultivation aims to create an artificial system mimicking the in situ conditions.However, we do not have sufficient knowledge to reproduce the natural conditions in the laboratory or to create viable synthetic conditions for 
all organisms. This lack of knowledge has led to regrettable mistakes.. For example, traces of tungsten element, which inactivates nitrogenase, have been included for a long time in the culture media for Spirochaetes (Leadbetter, 2003). It was recently discovered that Spirochaetes are able to grow via nitrogen fixation (Lilburn et al., 2001), a property that can be expressed only in tungsten-depleted media.Another remarkable example is the unexpected discovery of nanoarchaea in enrichment cultures (Huber et al., 2002).The discovery was unattainable by current PCR-based approaches since the universal primers commonly used for diversity studies could not target this group.

Another reason that can explain the failure to cultivate many prokaryotes is our lack of patience and sensitive detection methods for low cell yields. As previously discussed, in some cases, cultivation efficiency was significantly increased by long periods of incubation. This is true for organisms originating from oligotrophic habitats and potentially in a non-growing or dormancy state. It is important to note that transition from a non-growing to a growing state in a synthetic laboratory medium is a critical and stressful event. Thus, adaptations of cells to laboratory growth conditions may require increased cultivation times. For instance, the duration of growth log phase can depend on the cell status (i.e. healthy, stressed or sublethally damaged cells) and the gap between in situ and in vitro conditions (de novo synthesis of an enzymatic set to grow in the synthetic medium, etc). Without permitting adequate time intervals for growth, many organisms and many novel redox reactions would never have been discovered. For example, without lengthy incubation periods, the observation that methane is formed from long-chain alkanes under anaerobic conditions would not have been documented. Under tested conditions, gas formation in the presence of hexadecane started only after four-month incubation of the culture (Zengler et al., 1999). While bioenergetics calculations can predict thermodynamical feasibility of a reaction, our current knowledge can not predict kinetics for 'resuscitation' and for the growth of de novo enrichment cultures via a 
given reaction under a defined experimental conditions. Indeed, the characteristics of natural uncultured organism(s) (physico-chemical optima for growth, transport systems, etc.) and of their enzymes (catalytic rates, substrate affinities, regulation mechanisms) are unknown. At best, we can suppose that enrichment cultures performed with highly stable substrates, weak oxidants and calculated to have low net free energy gain, will require long-term incubations and patience. Otherwise, extremely long growth kinetics or low cell densities are sometimes observed in enrichment or pure culture. Inappropriate or non-optimal growth conditions might be responsible for these low yields. Thus, once parameters that inhibit growth are identified, "normal" growth kinetics and high yields can be restored (i.e. Flagan et al., 2003). For the organisms characterized by meagre yields, highly sensitive detection technologies such as tangential flow filtration and concentration are critical (Giovannoni and Stingl, 2007).

Another rational put forward for prokaryotic resistance to cultivation is inherent to the in vitro cultivation techniques widely used. More specifically, the in vitro cultivation techniques used paradoxically aim to isolate strains in pure culture, while most organisms in nature live in community and establish complex relationships (see previous chapter). The main consequence of this general practice is a disruption of inter- and intra-species communication during the very first stageof isolation. The impact of this perturbation on cultivability is unknown for most prokaryotic species and might vary depending of the species. Cell-cell communication (CCC) has gained considerable attention in recent years, in particular densitydependent cell-signalling mechanisms known as quorum sensing (QS). Cell-cell interactions have been investigated mainly in Bacteria due to their tremendous importance in health, environmental and industrial applications. Bacteria respond to a wide range of signalling molecules at intra-species level (species-specific compounds) and/or inter-species level (Camilli and Bassler, 2006). Inter-species interactions appear to be ubiquitous among prokaryotes and are not limited to signalling molecules such as autoinducer-2 (AI-2) and $N$ - 
acyl-homoserine lactones ( $N$-AHLs) but extend to antibiotics at sub-inhibitory concentrations (Ryan and Dow, 2008). Globally, prokaryotes and eukaryotes have co-existed for millions of years and frequently have co-evolved in the same environments. Therefore it is not surprising that interactions between them range from mutually beneficial to virulent. QS compounds like AHLs found initially in Bacteria are involved in virulence, biofilm formation, motility, antibiotic production and are recognized by eukaryotes with effects on immunomodulation, intracellular calcium signalling and apoptosis (Hughes and Speriandio, 2008). QS mechanisms have been extensively investigated during the past decade. Many Bacteria have one QS circuit while some display two or three circuits to coordinate their population density. Yet, these circuits are themselves under the control of a master QS regulator (Hooshangi and Bentley 2008). Recently Kolodkin-Gal et al. (2007, 2008) showed that the mode of action of antibiotics in E. coli is determined by the ability to communicate through the Extracellular Death Factor (EDF) as a function of cell density. CCC was initially considered as an exception limited to a few specialized bacteria, but has recently emerged as the norm in the bacterial world. Several indications strengthen this trend: (i) the high frequency of QS among genome-sequenced bacteria (40\% of 800 sequenced bacterial genomes contain the luxS gene suggesting that the Al-2 precursor functions as a universal signal (Pereira et al., 2008)) and, (ii) the discovery of signalling molecules in microbial metagenomic data. In addition, there is growing evidence that QS signals provide to bacteria more information than cell density. Surprisingly, the abundance of results on QS, biofilms and on cell-cell communication, at intra-species or inter-species levels have been analysed in the perspective of pathology (virulence, biofilm formation and control), biotechnology, synthetic biology (Hooshangi and Bentley, 2008), evolution (Keller and Surette, 2006; West et al., 2007), ecology (Nadell et al., 2008) but seldom for cultivation purposes. 
To account for CCC mechanisms in cultivation procedures remains a challenge due to our lack of knowledge on the cell-cell interaction requirements of targeted species. Considering the small fraction of prokaryotes cultivated, it seems likely that most cell-cell communication mechanisms are unknown. CCC mechanisms described up to now, like those classically observed with Vibrio harveyi, Pseudomonas aeruginosa and Escherichia coli, operate at high cell densities. We cannot exclude that some CCC mechanisms could also act at low cell densities $\left(10^{2}\right.$ to $10^{4}$ cells $\left./ \mathrm{ml}\right)$ to regulate cell growth of some species. In that case, it may be necessary to reconsider isolation procedures accordingly.

Finally, one last reason for which prokaryotes remain uncultured is due to enrichmentisolation process whereby the abiotic interactions are broken down. This disruption of the biogeochemical factors, that collectively represent the environment, is a source of stress for the organisms from natural habitats. The depreciation of these bio-geochemical factors from the native extra-cellular habitats leads the experimentalists to design synthetic conditions which might introduce a stressful parameter or a main change in resource type or concentration. For example, a phenomenon similar to substrate-accelerated death might occur. Growth inhibition is observed when cells are suddenly grown in the presence of concentrations greater than 1-10 $\mu \mathrm{M}$ of a given substrate (i.e. ammonia, phosphate or glucose) whereas they were previously starved of this substrate (Overmann, 2006).

\section{III- Cultivating the uncultured: future directions}

There is current growing consensus among microbiologists that improvements are needed in microbial cultivation. A comprehensive understanding of the microbial world will undoubtedly require cultivation. It is clear that the keys of cultivation will not come from a single technological breakthrough but will depend on our knowledge of the natural microbial systems. The numerous examples of successes in cultivation that we have mentioned in this 
review demonstrate that through patience and advances in technology, cultivation is a surmountable obstacle for many organisms. We still have a lot to learn from pure culture microbiology, even ifthese isolates might display unnatural behaviours in the synthetic laboratory.. Pure culture microbiology still represents the best method to study microbial physiology including detailed investigations on the role of genes, proteins, and metabolic pathways.. This is true since several unexpected physiological discoveries were made after isolation of novel species belonging to taxa presumed to be already well documented. In turn, these data provide important guidance for the optimization of cultivation media.

While several difficulties remain in cultivating microorganisms, future directions can be summarized as follows: refining culture medium, mimicking nature through in situ cultivation systems or designing devices supporting CCC, and developing automated procedures through robotics. Undoubtedly, combinations of these diverse approaches will yield successes in cultivation.

\section{1- Refinement of culture media}

Culture medium optimization will require the use of various complementary tools including: (i) the ability to define the range of electron donors, acceptors and key elements sources based on a better knowledge of the environment, (ii) molecular probes for screening novel species and, (iii) high-sensitive methods with low detection thresholds to uncover rare and slowgrowing species in culture. In the future, results from single cell genomics and metagenomics analyses will contribute to better isolation strategies for prokaryotes.

\section{2- Design of isolation strategies based on cell-cell interactions}

As seen previously, microbial landscapes are dominated by biofilms or aggregates. The isolation is not the preferred state of most Bacteria and Archaea. Despite the growing 
knowledge on cell-cell signalling molecules, the use of antibiotics and auto-inducers in culture is limited. Indeed, the vast majority of these compounds were characterized from microorganisms in pure culture, some of which were subsequently retrieved in metagenomic analyses. Metagenomics will yield numerous genes coding for novel auto-inducers or their precursors, but they will be classified as unknown genes until discovered in culture. Therefore it is not surprising that our previous analytical knowledge is hardly applicable to isolation procedures of novel species. For isolation of a strain, one way to preserve the endogenous CCC mechanisms is to grow itin microbial community conditions, using bioreactors, either under planktonic stage, or incorporated in gel microdroplets (GMDs) (Zengler et al., 2002), or attached to a surface. So far, the community culture step is more often followed by an isolation step through various means: streaking, dilution-to-extinction, sorting (mechanically or by FACS). For the last method, single cells are distributed in wells or micro-wells filled with appropriate medium and incubated for further culture. The major drawback is that the appropriate medium used to grow the cells after the isolation step is different from the medium that supported growth in the community culture, and that all chemical CCC are suddenly disrupted. What is the impact on the cultivability of some strains? In order to address this question it seems necessary to design novel culture equipments. The easiest method would be to substitute culture microplates by micro-bioreactors in the Zengler's method (Figure 3). In such a system, the flow from the community culture would nourish each well of the micro-bioreactor with all the metabolites produced by the community culture. This system would combine community culture and isolation. Further, it would allow for the circulation of chemical compounds in the micro-bioreactor, partly preserving cell-cell communication, even though physical cell-cell contacts would be disrupted. Since the community culture would operate as a black box for signalling compounds, we cannot exclude that some metabolites could have inhibitory effects. The benefits of such a 
combination of enrichment culture and isolation remain to be assessed. By dissociating and, in a following step, combining community culture and isolation, this system could address the culture of consortia components and symbionts which are almost impossible to solve with current practices.

\section{3- High throughput issues}

A quiet revolution occurred during the past decade in liquid handling applied to life sciences with the development of pipetting workstations. The main line of action was the reproduction at high throughput of manual procedures by pipetting robots. Culture medium distribution in micro-plate wells and inoculation of a single cell or GMD per well is now performed routinely by robots. The combination of GMD encapsulation and flow cytometry sorting is effective for aerobes (Akselband et al., 2006). Further, these studies show that some cells remain viable after staining and sorting, however these findings await confirmation. This remains a challenge for obligatory anaerobes especially due to the sorting step with flow cytometers. FACS equipment could be adapted to operate in anaerobic chambers, but might prove inconvenient and costly. The development of direct sorting of GMDs on liquid handling workstations would be an excellent alternative to avoid FACS analysis, to limit the cost of equipments and, to allow dissemination of these approaches in microbiology laboratories. What seems also to be certain is that the widespread use of high-throughput cultivation procedures implies the simultaneous development of affordable high-throughput identification procedures.

\section{4-Combination principle}

Microbiologists have long recognized that our understanding of the microbial world critically depends on the technological advances that broaden the knowledge-base for chemical, 
biological and physical processes. Today, it is established that an integration of the knowledge from multiple hierarchical levels of organization and from the molecule to the ecosystem are also required. Despite these facts, most efforts in cultivation focused only in the optimization of a limited set of parameters. We are convinced that this situation will change in the near future for several reasons. First, a consensus is gradually emerging: culture is not only needed to describe randomly novel species as a function of researcher main field of interest. Culture appears more and more as the sine qua non condition for understanding how the microbial world functions and is a prerequisite to predict changes in the frame of global change. Secondly, it seems that the complexity of the microbial world emerging from the growing knowledge of genomic and proteomic advances can not be analyzed only by combining these approaches and relying on systems biology. Finally, the advent of automation in culture and the combination of innovative methods lead to a new era in cultivation, assuming that some specific equipments are developed to handle the cell-cell interaction mechanisms during the clonal culture phase. Combinations theoretically possible are detailed in Fig. 3.

Ecosystems encompass abiotic conditions, living species and all their interactions (resource limitations, competition, predation, parasitism, among others). It is obvious that any cultivation attempt is by nature highly reductive and cannot reproduce the conditions observed in nature. The combination of existing methods and the development of novel approaches will help to come close to conditions where a significant fraction of microbes is amenable to culture (Fig. 2).

\section{Concluding remarks}


The extent of microbial diversity is still unknown and prokaryotes have undoubtedly many secrets to reveal. The success of microbial life on Earth stems from its great physiological diversity that collectively allows prokaryotes to derive energy from a wide range of redox reactions and to colonize several types of habitats; including the extreme ones. It is remarkable how much we have learned about microbial life by studying only a small fraction of its diversity. New examples of microbial ingenuity were recently discovered due to cultivation, either independently or in combination with metagenomics. There are still important essentials hidden in the $99.9 \%$ of the microbial world that are not yet cultured. They might change our understanding of biochemical processes, redox reactions, physiological adaptations and microbial behaviours. We postulate that in the future major advances in the understanding of microbial life will be achieved by innovative approaches in cultivation, but not cultivation alone. Coordinated efforts of researchers studying microbial systems at different levels should guide us to rethink culture strategies and to design growth conditions as close as possible to the natural interactions and conditions. In conclusion, the different cultivation successes discussed in this review demonstrate that contrary to widely held beliefs, many prokaryotes can be cultivated. In view of the gap between the number of phylotypes with or without representative cultured species, an additional question arises: is there a need to define relative priorities between phylotypes? The division amongst microbiologists at the international realm suggests that a consensus is not possible. The only consensus that could emerge, and successfully funded, is the need to investigate novel cultivation approaches, to develop new equipments and bring microbial cultivation in the $21^{\text {st }}$ century as a technologically advanced and a data rich discipline.

\section{ACKNOWLEDGEMENTS}

This work was funded by the Région Bretagne for the CPER Souchothèque and the joint research unit UMR6197, linking the Université de Bretagne Occidentale, the Ifremer and the Centre National de la 
Recherche Scientifique. We thank Stéphane L’Haridon for constructive discussions and critical reading of this manuscript.

\section{REFERENCES}

Achtman, M., Wagner, M. (2008) Microbial diversity and the genetic nature of microbial species. Nature Rev Microbiol 6: 431-440

Akselband, Y., Cabral, C., Castor, T.P., Chikarmane, H.M., Mc Grath, P. (2006). Enrichment of slowgrowing marine microorganisms from mixed cultures using gel microdrop (GMD) growth assay and fluorescence-activated cell sorting. J Exp Mar Biol Ecol 329: 196-205

Alain, K., Marteinsson, V.T., Miroshnichenko, M.L., Bonch-Osmolovskaya, E.A., Prieur, D., Birrien, J.-L. (2002) Marinitoga piezophila sp. nov., a rod-shaped, thermo-piezophilic bacterium isolated under high hydrostatic pressure from a deep-sea hydrothermal vent. Int J Syst Evol Microbiol 52: 1331-1339

Alain, K., Zbinden, M., Le Bris, N., Lesongeur, F., Querellou, J., Gaill, F., Cambon-Bonavita, M.-A. (2004) Early steps in microbial colonization processes at deep-sea hydrothermal vents. Environ Microbiol 6: 227-241

Amann, R.I., Ludwig, W., Schleifer, K.-H. (1995) Phylogenetic identification and in situ detection of individual microbial cells without cultivation. Microbiol Rev 59: 143-169

Araki, N., Ohashi, A., Machdar, I., Harada, H. (1999) Behaviors of nitrifiers in a novel biofilm reactor employing hanging sponge cubes as attachment sites. Wat Sci Tech 39: 23-31

Bollmann, A., Lewis, K., Epstein, S.S. (2007) Incubation of environmental samples in a diffusion chamber increases the diversity of recovered isolates. Appl Environ Microbiol 73: 6386-6390.

Brenner, K., you, L., Arnold, F.H. (2008) Engineering microbial consortia: a new frontier in synthetic biology. Trends in Biotechnol 26: 483-489

Bruns, A., Cypionka, H., Overmann, J. (2002) Cyclic AMP and acyl homoserine lactones increase the cultivation efficiency of heterotrophic bacteria from the central Baltic Sea. Appl Environ Microbiol 68: 3978-3987 
Bruns, A., Nübel, U., Cypionka, H., Overmann, J. (2003) Effect of signal compounds and incubation conditions on the culturability of freshwater bacterioplankton. Appl Environ Microbiol 69: 1980-1989

Button, D.K., Schut, F., Quang, P., Martin, R., Robertson, B.R. (1993) Viability and isolation of marine bacteria by dilution culture: theory, procedures, and initial results. Appl Environ Microbiol 59: 881-891

Camilli, A., Bassler, B.L. (2006) Bacterial small-molecule signaling pathways. Science 311: 11131116

Coates, J.D., Chakraborty, R., Lack, J.G., O’Connor, S.M., Cole, K.A., Bender, K.S., Achenbach, L.A. (2001) Anaerobic benzene oxidation coupled to nitrate reduction in pure culture by two strains of Dechloromonas. Nature 411: 1039-1043

Connon, S.A., Giovannoni, S.J. (2002) High-throughput methods for culturing microorganisms in very-low-nutrient media yield diverse new many isolates. Appl Environ Microbiol 68: 38783885

Crocetti, G.R., Banfield, J.F., Keller, J., Bond, P.L., Blackall, L.L. (2002) Glycogen-accumulating organisms in laboratory-scale and full-scale wastewater treatment processes. Microbiology 148: 3353-3364

Curtis, T.P., Sloan, W.T., Scannell, J.W. (2002) Estimating prokaryotic diversity and its limits. Proc Natl Acad Sci USA 99: 10494-10499

Davis, K.E.R., Joseph, S.J., Janssen, P.H. (2005) Effects of growth, inoculum size, and incubation time on culturability and isolation of soil bacteria. Appl Environ Microbiol 71: 826-834

Emerson, D., Moyer, C. (1997) Isolation and characterization of novel iron-oxidizing bacteria that grow at circumneutral pH. Appl Environ Microbiol 63: 4784-4792

Ferrari, B., Binnerup, S.J., Gillings, M. (2005) Microcolony cultivation on a soil substrate membrane system selects for previously uncultured soil bacteria. Appl Environ Microbiol 71: 8714-8720.

Ferrari, B.C., Winsley, T., Gillings, M., Binnerup, S. (2008) Cultivating previously uncultured soil bacteria using a soil substrate membrane system. Nature protocols 3: 1261-1269 
Flagan, S., Ching, W.-K., Leadbetter, J.R. (2003) Arthrobacter strain VAI-A utilizes acyl-homoserine lactone inactivation products and stimulates quorum signal biodegradation by Variovorax paradoxus. Appl Environ Microbiol 69: 909-916

Fröhlich, J., König, H. (2000) New techniques for isolation of single prokaryotic cells. FEMS Microbiol Rev 24: 567-572

Galushko, A., Minz, D., Schink, B., Widdel, F. (1999) Anaerobic degradation of naphthalene by a pure culture of a novel type of marine sulfate-reducing bacterium. Environ Microbiol 1: 415420

Gefen, O, Balaban, NQ (2008) The Moore’s law of microbiology - towards bacterial culture miniaturization with the micro-Petri chip. Trends Biotechnol 26: 345-347

Giovannoni S., Stingl, U. (2007) The importance of culturing bacterioplankton in the 'omics' age. Nature Rev Microbiol 5: 820-826

Hooshanghi, S., Bentley, W.E. (2008) From unicellular properties to multicellular behavior: bacteria quorum sensing circuitry and applications. Current opinion Biotechnol 19: 550-555

Houghton, J.L., Seyfried Jr W.E., Banta, A.B., Reysenbach, A.-L. (2007) Continuous enrichment culturing of thermophiles under sulfate and nitrate-reducing conditions and at deep-sea hydrostatic pressures. Extremophiles 11: 371-382

Huber, R., Burggraf, S., Mayer, T., Barns, S.M., Rossnagel, P., Stetter, K.O. (1995) Isolation of a hyperthermophilic archaeum predicted by in situ RNA analysis. Nature 376: 57-58

Huber, R., Huber, H., Stetter, K.O. (2000) Towards the ecology of hyperthermophiles: biotopes, new isolation strategies and novel metabolic properties. FEMS Microbiol Rev 24: 615-623

Huber, H., Hohn, M.J., Rachel, R., Fuchs, T., Wimmer, V.C., Stetter, K.O. (2002) A new phylum of Archaea represented by a nanosized hyperthermophilic symbiont. Nature 417: 63-67

Hugenholtz, P., Goebel, B.M., Pace, N.R. (1998) Impact of culture-independent studies on the emerging phylogenetic view of bacterial diversity. J Bacteriol 180: 4765-4774

Hugenholtz, P., Tyson, G.W. (2008) Metagenomics. Nature 455:481-483 
Hughes, D.T., Sperandio, V. (2008) Inter-kingdom signalling: communication between bacteria and their hosts. Nat Rev Microbiol 6: 111-120

Ingham, C.J., Sprenkels, A., Bomer, J., Molenaar, D., van den Berg, A., van Hylckama Vlieg, J.E.T., de Vos, W.M. (2007) The micro-Petri dish, a million-well growth chip for the culture and highthroughput screening of microorganisms. Proc Natl Acad Sci USA 104(46): 18217-18222

Janssen, P.H., Schuhmann, A., Mörschel, E., Rainey, F.A. (1997) Novel anaerobic ultramicrobacteria belonging to the Verrucomicrobiales lineage of bacterial descent isolated by dilution culture from anoxic rice paddy soil. Appl Environ Microbiol 63: 1382-1388

Joseph, S.J., Hugenholtz, P., Sangwan, P., Osborne, C.A., Janssen, P.H. (2003) Laboratory cultivation of widespread and previously uncultured soil bacteria. Appl Environ Microbiol 69: 7210-7215

Kaeberlein, T., Lewis, K., Epstein, S.S. (2002) Isolating ,uncultivable’ microorganisms in pure culture in a simulated natural environment. Science 296: 1127-1129

Kashefi, K., Lovley, D.R. (2003) Extending the upper temperature limit for life. Science 301:934

Keller, L., Surette, M.G. (2006) Communication in bacteria: an ecological and evolutionary perspective. Nat Rev Microbiol 4: 249-258

Kogure, K., Simidu, U., Taga, N. (1979) A tentative direct microscopic method for counting living marine bacteria. Can J Microbiol 25: 415-420

Kolodkin-Gal, I., Hazan, R., Gaathon, A., Carmeli, S., Engelberg-Kulka, H. (2007) A linear pentapeptide is a quorum-sensing factor required for mazEF-mediated cell death in Escherichia coli. Science 318: 652-655

Kolodkin-Gal, I., Engelberg-Kulka, H. (2008) The Extracellular Death Factor: physiological and genetic factors influencing ist production and response in Escherichia coli. J Bacteriol 190: 3169-3175

Könneke, M., Bernhard, A.E., de la Torre, J.R., Walker, C.B., Waterbury, J.B., Stahl, D.A. (2005) Isolation of an autotrophic ammonia-oxidizing marine archaeon. Nature 437: 543-546

Köpke, B., Wilms, R., Engelen, B., Cypionka, H., Sass, H. (2005) Microbial diversity in coastal subsurface sediments: a cultivation approach using various electron acceptors and substrate gradients. Appl Environ Microbiol 71: 7819-7830 
Leadbetter, J.R., Schmidt, T.M., Graber, J.R., Breznak, J.A. (1999) Acetogenesis from $\mathrm{H}_{2}$ plus $\mathrm{CO}_{2}$ by Spirochetes from termite guts. Science 283:686-689

Leadbetter, J.R. (2003) Cultivation of recalcitrant microbes: cells are alive, well and revealing their secrets in the $21^{\text {st }}$ century laboratory. Curr Opinion Microbiol 6: 274-281

Lovley, D.R., Lonergan, D.J. (1990) Anaerobic oxidation of toluene, phenol, and p-cresol by the dissimilatory iron-reducing organism, GS-15. Appl Environ Microbiol 56: 1858-1864

Marteinsson, V.T., Birrien, J.-L., Reysenbach, A.-L., Vernet, M., Marie, D., Gambacorta, A., Messner, P., Sleytr, U.B., Prieur, D. (1999) Thermococcus barophilus sp. nov., a new barophilic and hyperthermophilic archaeon isolated under high hydrostatic pressure from a deep-sea hydrothermal vent. Int J Syst Bacteriol 49: 351-359

Musat, F., Widdel, F. (2008) Anaerobic degradation of benzene by a marine sulphate-reducingenrichment culture, and cell hybridization of the dominant phylotype. Environ Microbiol 10: 10-19

Nadell, C.D., Xavier, J.B., Levin, S.A., Foster, K.R. (2008) The evolution of quorum sensing in bacterial biofilms. PLoS Biol 6:e14

Nadell, C. D., Xavier, J. B., Foster, K. R. (2009) The sociobiology of biofilms. FEMS Microbiol Rev 33: 206-224

Nauhaus, K., Boetius, A., Krüger, M., Widdel, F. (2002) In vitro demonstration of anaerobic oxidation of methane coupled to sulphate reduction in a sediment from a marine gas hydrate area. Environ Microbiol 4: 296-305

Nelson, D.C., Jannasch, H.W. (1983) Chemoautotrophic growth of a marine Beggiatoa in sulfidegradient cultures. Arch Microbiol 136: 262-269 
Overmann, J (2006) Principles of Enrichment, Isolation, Cultivation and Preservation of Prokaryotes. In: Dworkin, M., Falkow, S., Rosenberg, E., Schleifer, K.-H., Stackebrandt, E. (eds). The Prokaryotes, third edition. Springer, New-York, pp 80-136

Pereira, C.S., Mc Auley, J.R., Taga, M.E., Xavier, K.B., Miller, S.T. (2008) Sinorhizobium meliloti, a bacterium lacking the autoinducer-2 (AI-2) synthase, responds to AI-2 supplied by other bacteria. Mol Microbiol 70: 1223-1235

Pignatelli, M., Aparicio, G., Blanquer, I., Hernandez, V., Moya, A., Tamames, J. (2008) Metagenomics reveals our incomplete knowledge of global diversity. Bioinformatics 24: 21242125

Plugge, C.M., Stams, A.J.M. (2002) Enrichment of thermophilic syntrophic anaerobic glutamatedegrading consortia using a dialysis membrane reactor. Microbial Ecol 43: 379-387

Pörtner, R., Märkl, H. (1998) Dialysis cultures. Appl Microbiol Biotechnol 50: 403-414

Postec, A., Urios, L., Lesongeur, F., Ollivier, B., Querellou, J., Godfroy, A. (2005) Continuous enrichment culture and molecular monitoring to investigate the microbial diversity of thermophiles inhabiting deep-sea hydrothermal ecosystems. Curr Microbiol 50: 138-144

Postec, A., Lesongeur, F., Pignet, P., Ollivier, B., Querellou, J., Godfroy, A. (2007) Continuous enrichment cultures : insights into prokaryotic diversity and metabolic interactions in deep-sea vent chimneys. Extremophiles $11: 747-757$

Rabus, R., Nordhaus, R., Ludwig, W., Widdel, F. (1993) Complete oxidation of toluene under strictly anoxic conditions by a new sulfate-reducing bacterium. Appl Environ Microbiol 59: 1444-1451

Raghoebarsing, A.A., Pol, A., van de Pas-Schoonen, K.T., Smolders, A.J.P., Ettwig, K.F., Rijpstra, W.I.C., Schouten, S., Sinninghe Damsté, J.S., Op den Camp, H.J.M, Jetten, M.S.M., Strous, M. (2006) A microbial consortium couples anaerobic methane oxidation to denitrification. Nature 440: 918-921

Rappé, M.S., Connon, S.A., Vergin, K.L., Giovannoni, S.J. (2002) Cultivation of the ubiquitous SAR11 marine bacterioplankton clade. Nature 418: 630-633 
Ryan, R.P., Dow, J.M. (2008) Diffusible signals and interspecies communication in bacteria. Microbiology 154: 1845-1858

Sait, M., Hugenholtz, P., Janssen, P.H. (2002) Cultivation of globally distributed soil bacteria from phylogenetic lineages previously only detected in cultivation-independent surveys. Environ Microbiol 4: 654-666

Sangwan, P., Kovac, S., Davis, K.E.R., Sait, M., Janssen, P.H. (2005) Detection and cultivation of soil Verrucomicrobia. Appl Environ Microbiol 71: 8402-8410

Schink, B., Friedrich, M. (2000) Phosphite oxidation by sulphate reduction. Nature 406: 37

Schink, B. (1999) Ecophysiology and ecological niches of prokaryotes. In: Lengeler, J.W., Drews, G., Schlegel, H.G. (eds) Biology of the prokaryotes. Georg Thieme Verlag, Stuttgart, pp. 723-762

Skinner, F.A., Jones, P.C.T., Mollison, J.E. (1952) A comparison of a direct- and a plate-counting technique for the quantitative estimation of soil micro-organisms. J Gen Microbiol 6: 261-271

Staley, J.T., Konopka, A. (1985) Measurement of in situ activities of nonphotosynthetic microorganisms in aquatic and terrestrial habitats. Annu Rev Microbiol 39: 321-346

Stevenson, B.S., Eichorst, S.A., Wertz, J.T., Schmidt, T.M., Breznak, J.A (2004) New strategies for cultivation and detection of previously uncultured microbes. Appl Environ Microbiol 70: 47484755

Stingl, U., Tripp, H.J., Giovannoni, S.J. (2007) Improvements of high-throughput culturing yielded novel SAR11 strains and other abundant marine bacteria from the Oregon coast and the Bermuda Atlantic Time Series study site. The ISME J 1: 361-371

Stott, M.B., Crowe, M.A., Mountain, B.W., Smirnova, A.V., Hou, S., Alam, M., Dunfield, P.F. (2008) Isolation of novel bacteria, including a candidate division, from geothermal soils in New Zealand. Environ Microbiol 10: 2030-2041

Takai, K., Nakamura, K., Toki, T., Tsunogai, U., Miyazaki, M., Miyazaki, J., Hirayama, H., Nakagawa, S., Nunoura, T., Horikoshi, K. (2008) Cell proliferation at $122^{\circ} \mathrm{C}$ and isotopically heavy $\mathrm{CH}_{4}$ production by a hyperthermophilic methanogen under high-pressure cultivation. Proc Natl Acad Sci USA 105: 10949-10954 
Tamaki, H., Sekiguchi, Y., Hanada, S., Nakamura, K., Nomura, N., Matsumura, M., Kamagata, Y. (2005) Comparative analysis of bacterial diversity in freshwater sediment of a shallow eutrophic lake by molecular and improved cultivation-based techniques. Appl Environ Microbiol 71: 2162-2169

Watve, M., Shejval, V., Sonawane, C., Rahalkar, M., Matapurkar, A., Shouche, Y., Patole, M., Phadnis, N., Champhenkar, A., Damle, K., Karandikar, S., Kshirsagar, V., Jog, M. (2000) The 'K' selected oligophilic bacteria: a key to uncultured diversity? Curr Sci 78: 1535-1542

West, S.A., Diggle, S.P., Buckling, A., Gardner, A., Griffin, A.S. (2007) The social lives of microbes. Annu Rev Ecol Evol Syst 38: 53-77

Whitman, W.B., Coleman, D.C., Wiebe, W.J. (1998) Prokaryotes: The unseen majority. Proc Natl Acad Sci USA 95: 6578-6583

Widdel, F., Musat, F., Knittel, K., Galushko, A. (2007) Anaerobic degradation of hydrocarbons with sulphate as electron acceptor. In Sulphate-reducing bacteria: Environmental and Engineered Systems. Barton, L.L., and Hamilton, W.A. (eds). Cambridge, UK: Cambridge University Press, chapter 9, pp. 265-303

Yasumoto-Hirose, M., Nishijima, M., Ngirchechol, M.K., Kanoh, K., Shizuri, Y., Miki, W. (2006) Isolation of marine bacteria by in situ culture on media-supplemented polyurethane foam. Mar Biotech 8: 227-237

Yayanos, A.A., Dietz, A.S., Van Boxtel, R. (1979) Isolation of a deep-sea barophilic bacterium and some of its growth characteristics. Science 205: 808-810

Yayanos, A.A., Dietz, A.S., Van Boxtel, R. (1981) Obligately barophilic bacterium from the Mariana Trench. Proc natl Acad Sci USA 78: 5212-5215

Zeng, X, Birrien, J-L, Fouquet, Y, Cherkashov, G, Jebbar, M, Querellou, J, Oger, P, Cambon-Bonavita, M-A, Xiao, X, Prieur, D. Pyrococcus CH1, an obligate piezophilic hyperthermophile: extending the upper pressure-temperature limits for life. ISME J (in press: doi:10.1038/ismej.2009.21) Zengler, K., Richnow, H.H., Rossello-Mora, R., Michaelis, W., Widdel, F. (1999) Methane formation from long-chain alkanes by anaerobic microorganisms. Nature 401:266-269 
2

Zengler, K., Toledo, G., Rappé, M., Elkins, J., Mathur, E.J., Short, J.M., Keller, M. (2002) Cultivating the uncultured. Proc Natl Acad Sci USA 99: 15681-15686

Zengler, K., Walcher, M., Clark, G., Haller, I., Toledo, G., Holland, T., Mathur, E.J., Woodnutt, G., Short, J.M., Keller, M. (2005) High-throughput cultivation of microorganisms using microcapsules. Methods in Enzymol 397: 124-130 


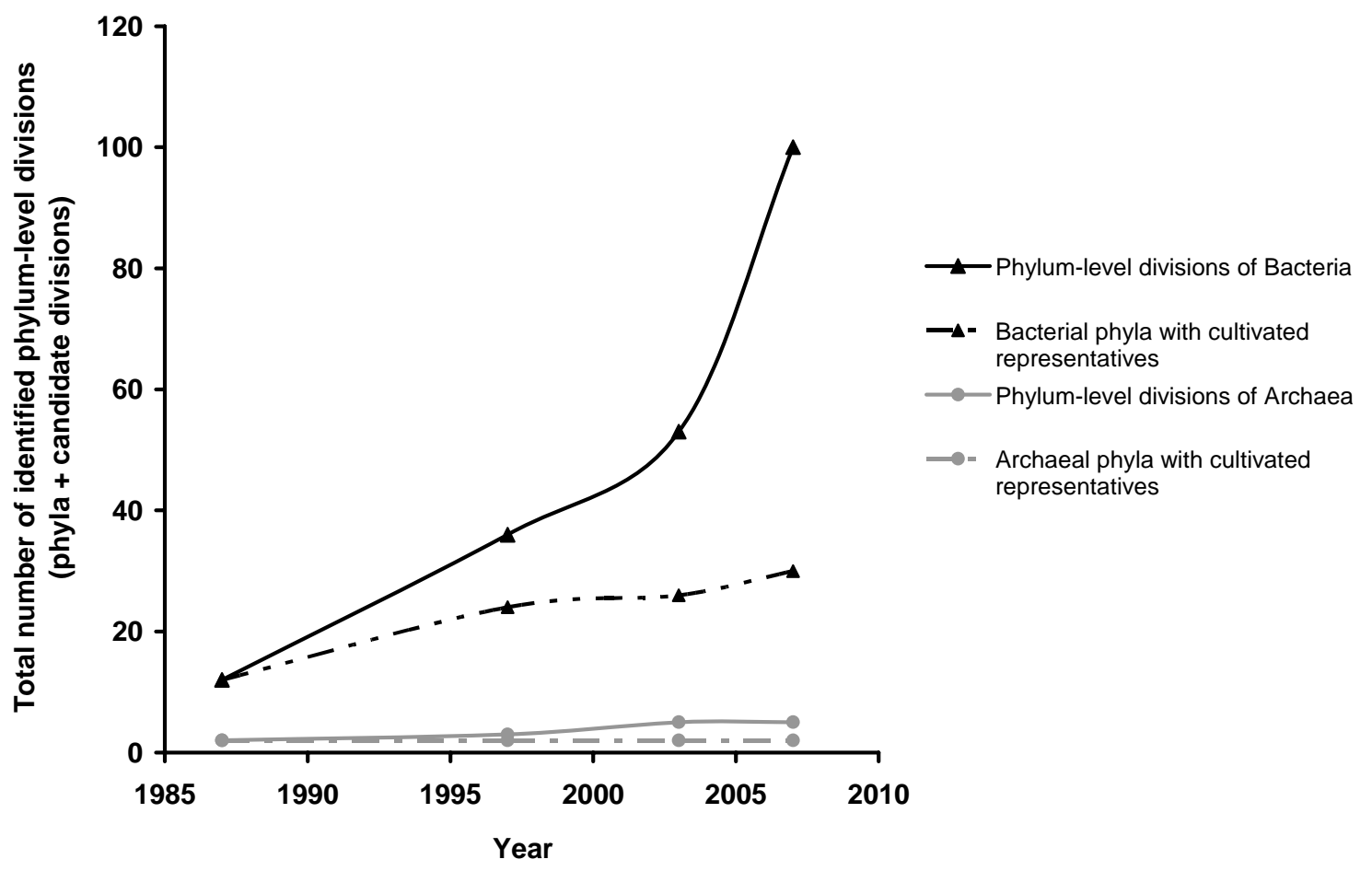

Fig. 1. Numbers of phylum-level divisions (phyla with cultivated members + candidate divisions with no cultivated representative) identified since 1987 among Bacteria (black line) and Archaea (grey line), and numbers of phyla with cultivated representatives (dotted lines). Adapted from Achtman \& Wagner (2008). 


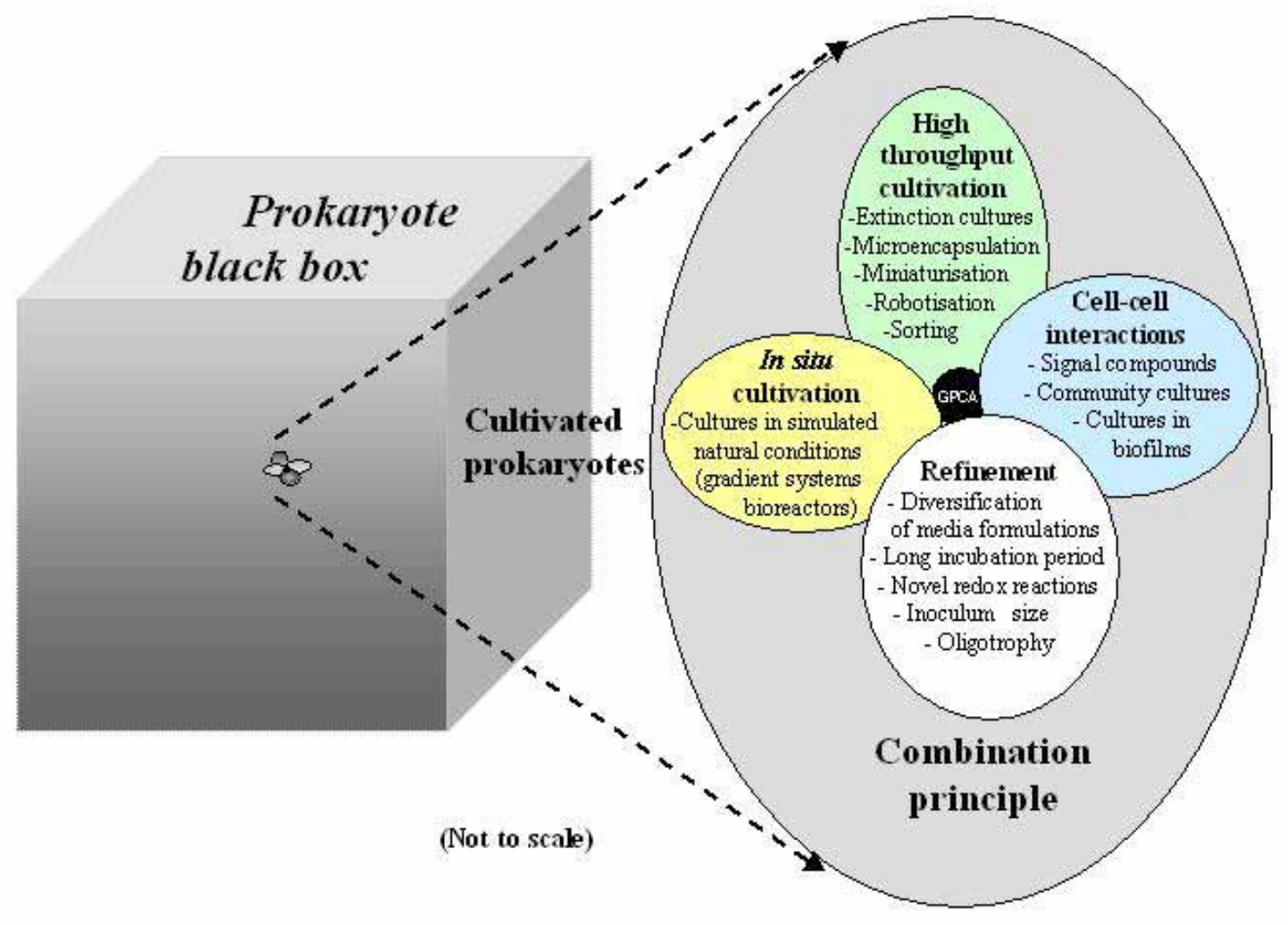

Fig. 2. Schematic representation of the four main categories of cultivation strategies. The small black circle schematizes the minor fraction of a naturally occurring microbial community that arerecovered onto conventional selective media, the so-called "Great Plate Count Anomaly”. Improved cultivation strategies developed so far aimto enhance one or few aspects of growth conditions among four main categories (represented by four bullets) and each allowed individually recovering more numerous and/or more diverse isolates than traditional approaches. None of these improved approaches is universal. We postulate that the combination ("the combination principle") of already existing methods belonging to these four categories will help to come close to conditions where a significant fraction of microbes is amenable to culture. 

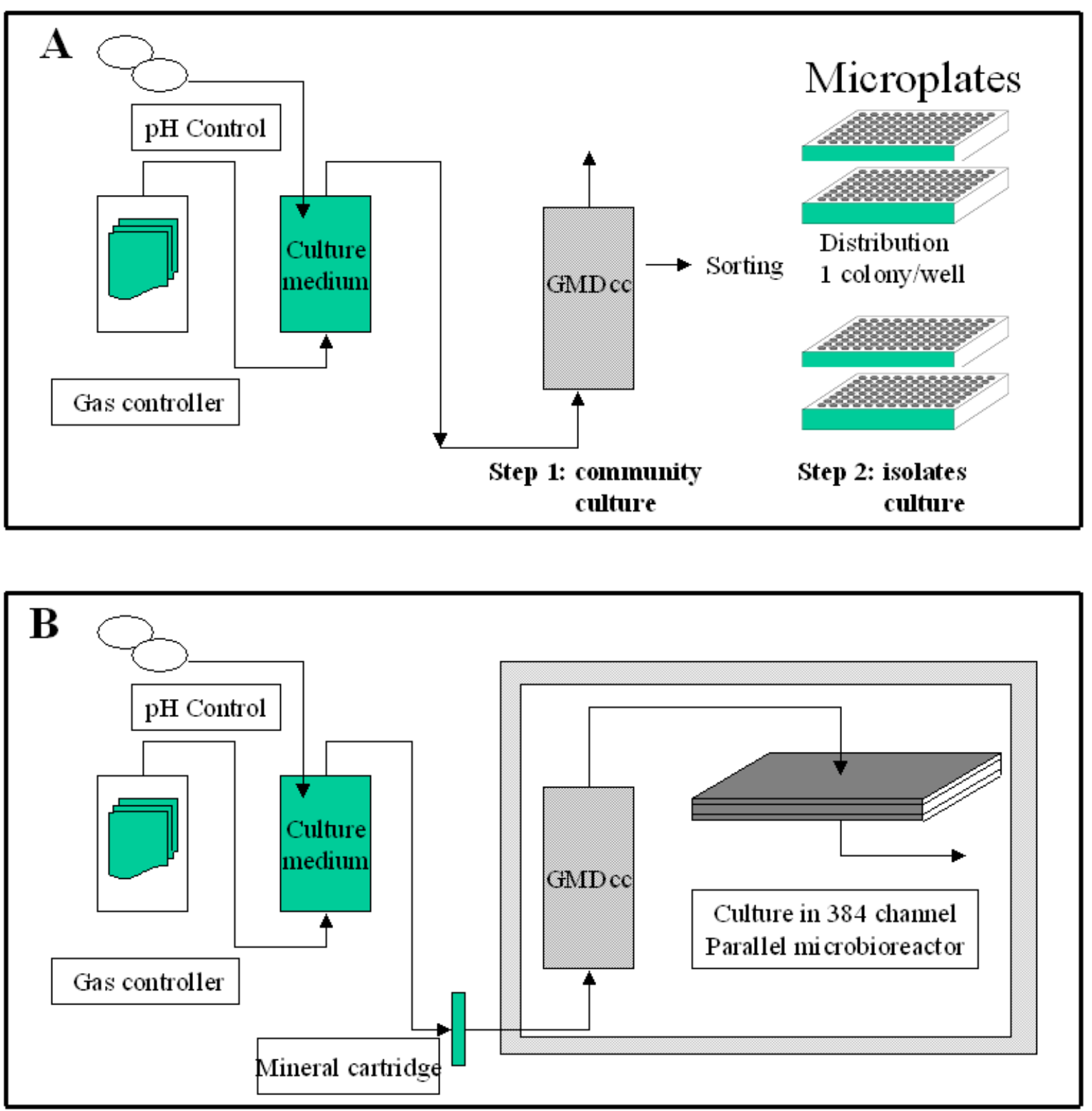

791

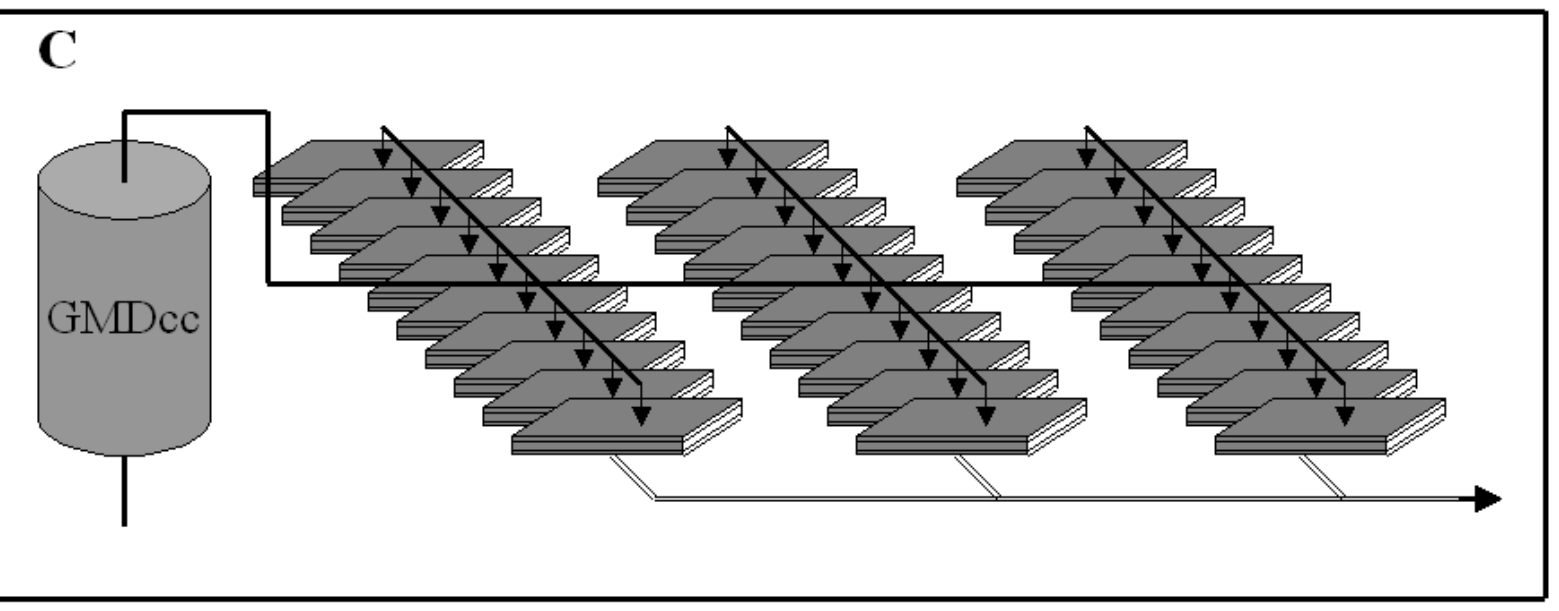


Fig. 3. Schematic diagram of cultures and isolation procedures based on the combination 794 principle.

796 droplets

797 A: GMD community culture followed by sorting and micro-plate cultivation (From Zengler et al., 2002, modified).

B: flowthrough culture in parallel micro-bioreactors nourished by community culture medium

800 and metabolite products. Micro-plates are replaced by micro-bioreactors directly connected to 801 the GMD community culture. This system can be operated in aerobic or anaerobic conditions. 802 Micro-bioreactors (either 96 or 384 parallel channels SBS format) and flowthrough 803 equipments awaiting development.

804 C: GMD and micro-bioreactors (384 channels) coupled in a nearly theoretical 10000 parallel 805 channels configuration. 